Research Paper:

\title{
The Relationship Between Illness Perception and Difficulty in Emotion Regulation with the Mediating Role of Quality of Marital Life in Women With Breast Cancer
}

\author{
Raheleh Firouzi $^{1}$, Taher Tizdast $^{1^{*}}$, Javad Khalatbari ${ }^{1}$, Shohreh Ghorban Shiroudi ${ }^{1}$
}

Citation Firouzi R, Tizdast T, Khalatbari J, Ghorban Shiroudi Sh. The Relationship Between Illness Perception and Difficulty in Emotion Regulation with the Mediating Role of Quality of Marital Life in Women With Breast Cancer. Avicenna J of Neuropsychophysiology. 2019; 6(4):185-194. http://dx.doi.org/10.32598/ajnpp.6.4.4

\section{(A) 03}

Article info:

Received: 18 May 2019

Accepted: 05 Sep 2019

Available Online: 01 Nov 2019

Keywords:

Illness Perception, Emotion Regulation, Marital Life Quality, Breast Cancer

\section{A B S T R A C T}

Background: The aim of this research was to investigate the relationship between perception of illness and difficulty in emotion regulation with the mediating role of quality of marital life in married women with breast cancer.

Objectives: It is very important to study The Relationship Between IIIness Perception and Difficulty in Emotion Regulation with the Mediating Role of Quality of Marital Life in Women With Breast Cancer.

Materials and Methods: This correlational study using a structural equation modeling was conducted on 385 married women suffering from breast cancer who referred to the Amol and Babol medical centers in 2018. The samples were selected based on the available sampling method and completed IIIness Perception Questionnaire (IPQ), Difficulty in Emotion Regulation Scale (DERS), and Marital Quality of Life Questionnaire. The proposed model was evaluated by structural equation modeling using LISREL software. The bootstrap method was used to test indirectly.

Results: Based on the results of this study, the proposed model had a good fitness (RMSEA $=0.057, \mathrm{GFI}=0.92, \mathrm{IFI}=0.98, \chi^{2}=0.225$, and $\mathrm{P}<0.05$ ). Generally, the results showed that all direct pathways were significant $(P<0.05)$.

Conclusion: The results also showed that indirect pathways of illness perception through marital life quality with difficulty in emotion regulation were significant. The evaluated model had a good fit that is an important step in understanding the factors affecting the difficulty in emotion regulation in married women with breast cancer. Therefore, it can be useful as a model for designing and developing programs for the prevention of emotional problems in women with breast cancer.

\section{* Corresponding Author:}

Taher Tizdast, $P h D$.

Address: Department of Psychology, Islamic Azad University, Tonekabon Branch, Tonekabon, Iran.

Tel: +98 (912) 3175368

E-mail: tahertizdast@yahoo.com 


\section{Introduction}

B

reast cancer is the most common, the emotionally and psychologically deadliest, the most influential, and the most important health concern among women. Breast cancer is an uncontrollable growth of a series of abnormal cells in the breast area that can invade surrounding tissue and spread through lymphatic channels and bloodstream. Living with chronic pain resulting from cancer requires tolerating significant emotional stress. Also, pain reduces affective and emotional abilities, which ultimately weakens the patient's morale, causes feelings of hopelessness, frustration, and depression, and declines the quality of life. Emotional theories emphasize how regulating an emotional situation, such as a trauma or sudden physical illness, plays an important role in the emotional response to that event [1].

Difficulty in emotion regulation is not a single and homogeneous process, but it is realized through different mechanisms. Difficulty in regulating emotions in different individuals requires activation of some cognitive processes that make the person adopt difficult strategies in regulating different and specific emotions [2]. Regulation of poor emotion regulation or lack of emotional expression can predict premature death due to cancer. Emotional repression tendencies were predicted by breast cancer [3].

On the other hand, illness perceptions include some dimensions, such as timeline: (acute/chronic and cyclical), illness consequences, personal control, treatment control, illness coherence, and emotional representations [4]. In other words, illness perception refers to the person's general perception regarding his illness and indicates his ability to attribute various symptoms of the disease to his illness and to the extent he has developed negative emotional reactions, such as fear [5]. On the other hand, people di- agnosed with breast cancer develop symptoms of depression and anxiety, which can lower their quality of life [6].

Breast cancer in women has an adverse impact on their marital life and leads to a decline in marital life quality. Marital quality is an important aspect of family life that is associated with individuals' health and wellbeing [7] and is correlated with couples' physical and psychological health [8]. Also, quality of marital life is likely to be an important source of adult physical health, generally even among those without experiencing a chronic stressor, such as caring for a sick spouse [9].

This research was designed based on the Oh and Hwang [10] study aimed at examining the "relationship between uncertainty and quality of life in patients with breast cancer with the mediating role of marital intimacy". This study showed a significant relationship between uncertainty and quality of life in patients with breast cancer. Marital intimacy can also mediate the relationship between uncertainty and quality of life in patients with breast cancer. Due to the major role of the breast in the female sex, the response to this disease can include depression, anxiety, and stress. These reactions can make patients with breast cancer facing problems, such as sexual dysfunction, weakness in making therapeutic decisions, poor loyalty to treatment regimens, poor social interaction, weakening of marital life, and ultimately a reduction in quality of life. Accordingly, it raises a question to answer whether there is a relationship between the perception of illness and difficulty in emotion regulation with the mediating role of quality of marital life in married women with breast cancer.

\section{Materials and Methods}

The present descriptive correlational study was using the structural equation modeling was conducted on all married women with breast cancer referring to the Ary-

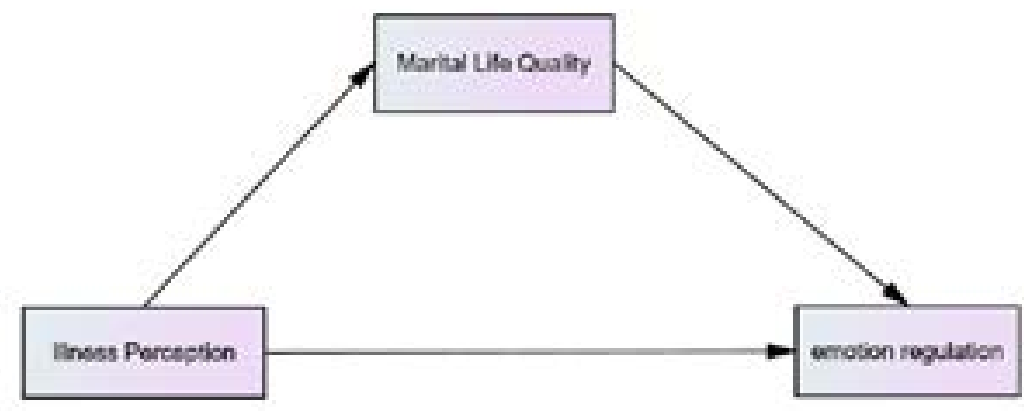

Figure 1. The conceptual model and structural equation modeling model 
an Clinic in Amol, Breast Cancer Diagnosis Association, and Medical Centers in Amol and Babol between November and March 2018. Since the sample size was unknown, using the Cochran formula, the sample size was estimated at 385 individuals selected using the available sampling method. The conceptual model and structural equation modeling model is presented in Figure 1.

The study protocol was approved by the Islamic Azad University, Tonekabon Branch. Inclusion criteria included the diagnosis of breast cancer by a specialist physician, a history of breast surgery, married women with breast cancer, no drug and psychotropic drugs use, and the exclusion criteria were informed consent to participate in the study, no breast cancer diagnosed by a specialist, no history of breast surgery, single women with breast cancer, and drug abuse.

\section{Illness Perception Questionnaire (IPQ)}

The revised IIIness Perception Questionnaire (IPQ-R) was developed by Moss-Morris et al. in 2002 [11]. It includes 44 items and measures 7 subscales of the timeline (acute/chronic), timeline (cyclical), consequences, personal control, treatment control, illness coherence, and emotional representation. It is rated on a 5-point Likert scale as follows: strongly disagree (1), disagree (2), neutral (3), agree (4), and strongly agree (5). The total score of the scale is from 40-220. Cronbach's alpha coefficient was used to investigate the internal consistency of the questionnaire, which obtained 0.71 [12]. In a study, the Cronbach's alpha for its components ranged from $0.63-0.83$, and the correlation coefficients through the test-retest for two weeks and six months ranged 0.62-0.91 and 0.53-0.88, respectively, significant at 0.01 level [13]. In recent research, the Cronbach's alpha coefficient of the questionnaire was $0.75-0.88$ [14]. In this research, to evaluate the reliability of the questionnaire using Cronbach's alpha coefficient, coefficients for the timeline (acute and chronic), timeline (cyclical illness), illness consequence, illness control, illness treatment, illness coherence, emotional representation, and the whole scale questions were $0.71,0.89,0.70,0.81,0.73$, 0.81 , and 0.71 , respectively.

\section{Difficulty in Emotions Regulation Scale (DERS)}

DERS was developed by Gratz and Roemer in 2004. It includes 36 items and measures 6 components of emotional response rejection, difficulty in using targeted behaviors, control difficulty, lack of emotional awareness, limited access to emotional regulation strategies, and lack of emotional clarity [15]. DERS is scored on a 5-point Likert scale ranging from almost never to almost always: Almost never (1), sometimes (2), in almost half of the cases (3), most often (4), and almost always (5), and the total score of the questionnaire ranges from 36-180 [16]. Using the Pearson correlation, the convergent validity was obtained 0.69 for depression, 0.36 for community-based perfectionism, and 0.24 for individual-centered perfectionism. The constructs validity using Pearson correlation was found 0.80 for non-acceptance of emotional responses, 0.67 for the difficulty in using targeted behaviors, 0.81 for difficulty controlling momentum, 0.40 for lack of emotional awareness, 0.88 for limited access to adjustment strategies emotionally, and 0.78 for lack of emotional clarity, indicating the construct validity [17]. In another study, Cronbach's alpha of the whole scale was 0.96 , indicating the high reliability of the DERS [18]. In this research, to investigate the reliability of the DERS its internal consistency using Cronbach's alpha, coefficients for rejecting emotional responses, difficulty in using targeted behaviors, impulsivity control difficulty, lack of emotional awareness, limited access to emotion regulation strategies, lack of emotional clarity, and total questions were 0.77, 0.79, $0.79,0.81,0.71,0.83$, and 0.84 , respectively.

\section{Marital Quality of Life Questionnaire}

The revised Marital Quality of Life Questionnaire was developed by Busby et al. in 1995 and included 14 items measuring three components of marital agreement, marital satisfaction, and marital coherence. It is scored on a 6-point Likert scale as follows: always $=6$, almost always $=5$, most often $=4$, usually $=3$, little $=2$, and rarely $=1$. In general, high scores indicate a higher quality of marital life [19]. Also, the internal consistency of the whole questionnaire using Cronbach's alpha coefficient in other countries was reported to be 0.86 [20]. In this study, to investigate the internal consistency reliability of the questionnaire using Cronbach's alpha, the coefficients for marital agreement, marital satisfaction, marital cohesion, and whole questionnaire were $0.81,0.80,0.74$, and 0.87 , respectively.

Theoretical data were collected using definitions and theories of the considered variables available in the studies indexed in the Iranian journals affiliated to the Ministry of Science, Ministry of Health, Islamic Azad University, and other journals affiliated to the scientific associations and regarding English articles, studies available in the Google Scholar databases were used. After collecting necessary data from the Aryan Clinic, Breast Cancer Diagnosis Association, clinics providing breast cancer diagnostic services, and specialists in Amol 
and Babol in 2018, and referring to these centers, the samples were selected. Married women referring to the clinics and physicians' offices for chemotherapy, radiation therapy, or controlling their course of treatment were identified and those willing to participate in the research signed the consent form. The consent form included the ethics of the research, describing the free and voluntary participation and the confidentiality of the answers. Then, the research questionnaires were distributed and the subjects were asked to read all the questions carefully and do not leave questions answered as much as possible. The researcher supervised the process of answering the questionnaires until all questionnaires were completed and no questionnaires remained unanswered.

They were also informed that as an acknowledgment for their participation in the research, those who wish to be informed about the results of their tests could write their names or nicknames in the questionnaire Approximately half of the participants first completed the Questionnaires of Illness Perception, DERS, and Quality of Marital Life, whereas the other half answered in reverse to control the effects of order of completing the questionnaires. After data collection, the completed questionnaires were analyzed by appropriate statistical methods. According to the American Psychological Association, the rights of individuals participating in research and considering their human rights should be respected. Our samples were informed that participation in this research is not dangerous or it has the least risk.

Descriptive and inferential statistics were used to analyze the data. Regarding descriptive statistics, the central tendency Mean \pm SD were used. KolmogorovSmirnov test was used for assessing the normal distri- bution of data and the Watson-Durbin test for checking the assumption of independence of errors. The tolerance coefficient and variance inflation factor were also used for a lack of multi-linearity. Considering inferential statistics, the Pearson correlation coefficient and the structural equation model were used to investigate the relationships between variables using LISREL software. The direct effect of illness perception on quality of marital life is presented in Figure 2.

\section{Results}

One-sample Kolmogorov-Smirnov test showed that the distribution of scores of the research variables was 95\% normal ( $P>0.05)$; therefore, the distribution of the scores has a normal distribution. Demographic characteristics of the subjects are presented in Table 1. On this basis, it can be concluded that the distribution of their data is normal and natural. Also, since Watson-Durbin statistics are between 1.5 and 2.5, which indicates that the correlation between the errors is rejected, i.e., the errors are not correlated. Descriptive Statistics of research variables are presented in Table 2 . The values of the variance inflation factor also showed that none of the indices are much greater than 10 and are close to 10 , so there is no problem in using linear regression. The bootstrapping method was used in computer instruction of Preacher and Hayes' MACRO (2004) to investigate the indirect relationship between the perception of illness with difficulty in regulating emotion and mediating the role of marital life quality. The results of the bootstrapping method for examining indirect paths are presented in Table 3.

Table 3 shows the bootstrapping test of the indirect causal relationships of the research variables. Based on Table 3, if

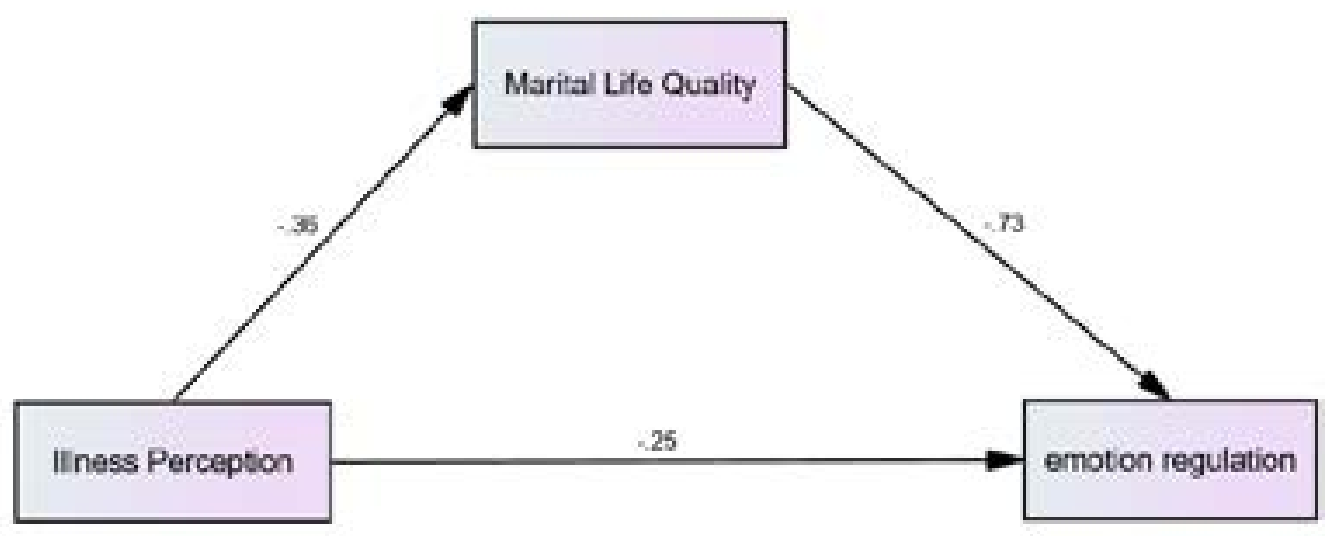

Figure 2. Path coefficients of the direct effect of illness perception on quality of marital life 
Table 1. Demographic characteristics of the subjects

\begin{tabular}{|c|c|c|}
\hline \multicolumn{2}{|c|}{ Demographic Variables } & \multirow{2}{*}{$\begin{array}{c}\text { Frequency (\%) } \\
27(7)\end{array}$} \\
\hline \multirow{6}{*}{ Age (year) } & $28-38$ & \\
\hline & $39-49$ & $103(26.8)$ \\
\hline & $50-60$ & $151(39.2)$ \\
\hline & $61-71$ & $97(25.2)$ \\
\hline & 72 and above & $7(1.8)$ \\
\hline & Total & $385(100)$ \\
\hline \multirow{5}{*}{ Education } & Lower than diploma & 267 (69.35) \\
\hline & Diploma & $77(20)$ \\
\hline & Associate diploma & 15 (3.9) \\
\hline & M.A. and above & $26(6.75)$ \\
\hline & Total & $385(100)$ \\
\hline \multirow{3}{*}{ Occupation } & Housewife & $267(69.35)$ \\
\hline & Employee & $118(30.65)$ \\
\hline & Total & $385(100)$ \\
\hline \multirow{3}{*}{ Illness duration (year) } & $1-5$ & $353(91.68)$ \\
\hline & $6-8$ & $32(8.31)$ \\
\hline & Total & $385(100)$ \\
\hline \multirow{5}{*}{ Duration of being married (year) } & $5-15$ & $33(8.6)$ \\
\hline & $16-26$ & $110(28.6)$ \\
\hline & $27-37$ & $133(34.5)$ \\
\hline & 38 and above & $109(28.31)$ \\
\hline & Total & $385(100)$ \\
\hline \multirow{3}{*}{ Number of children } & $1-3$ & $292(75.84)$ \\
\hline & $4-6$ & $93(24.16)$ \\
\hline & Total & $385(100)$ \\
\hline
\end{tabular}

the upper and lower limits of the bootstrapping index are in one direction, i.e., both are positive or negative, then it can be concluded that illness perception through marital life quality with difficulty in emotion regulation is indirect, and the indirect causal path is significant. Table 4 shows the fitness indices of the research model.

According to Table 4, the root mean square error of approximation (RMSEA) index was 0.057 , indicating a good and desirable fitness of the model. Therefore, the general hypothesis assuming the good fitness of the model of the relationship between illness perception and difficulty in emotion regulation with the mediating role of marital life quality was confirmed.

As it can be seen in Table 5, in the research model, the direct pathways of the effect of illness perception on quality of marital life $(\beta=-0.361, P$-value $=0.005)$ and difficulty in emotion regulation $(\beta=0.225$, $P$-value $=0.003$ ) were significant.

\section{Discussion}

The considered hypothesis assuming the good fitness of the model of the relationship between illness perception and difficulty in emotion regulation with the mediating role of marital life quality was confirmed. This result is consistent with that of Oh and Hwang [10] who indicated a significant relationship between uncertainty and quality of life in patients with breast cancer with the mediating role of marital intimacy.

Regarding the relationship between the perception of illness and the mediating role of quality of marital life over the difficulty in emotion regulation, the illness perception or cognitive representation of the illness is formed by the patient based on the information gained from different sources and beliefs. This factor can affect the patient's mental health and ability to adjust to the illness [21]. IIIness perception is an organized cognitive representation 
Table 2. Minimum, maximum, and Mean \pm SD of the research variables

\begin{tabular}{|c|c|c|c|}
\hline Variables & Min. & Max. & Mean $\pm S D$ \\
\hline Timeline (acute and chronic) & 11 & 26 & $18.83 \pm 5.704$ \\
\hline Timeline (cyclical illness) & 8 & 16 & $12.19 \pm 2.502$ \\
\hline Illness consequence & 11 & 25 & $17.70 \pm 5.164$ \\
\hline Personal control of illness & 12 & 28 & $20.28 \pm 5.858$ \\
\hline Treatment control & 9 & 17 & $12.44 \pm 2.496$ \\
\hline Illness coherence & 11 & 28 & $19.65 \pm 5.918$ \\
\hline Emotional representation & 11 & 28 & $19.98 \pm 5.467$ \\
\hline The total score of illness perception & 78 & 162 & $121.06 \pm 29.534$ \\
\hline Rejecting emotional responses & 14 & 33 & $22.78 \pm 7.314$ \\
\hline Difficulty in applying purposeful behaviors & 11 & 24 & $17.33 \pm 4.875$ \\
\hline Difficulty of impulsivity control & 10 & 23 & $16.95 \pm 4.302$ \\
\hline Lack of emotional awareness & 13 & 33 & $23.32 \pm 7.018$ \\
\hline Limited access to emotional regulation strategies & 14 & 36 & $23.93 \pm 9.478$ \\
\hline Lack emotional clarity & 10 & 22 & $16.34 \pm 4.117$ \\
\hline Total score of difficulty in emotional regulation & 78 & 168 & $120.65 \pm 32.354$ \\
\hline Marital agreement & 10 & 28 & $18.24 \pm 7.046$ \\
\hline Marital satisfaction & 10 & 27 & $17.48 \pm 6.323$ \\
\hline Marital cohesion & 4 & 15 & $9.29 \pm 2.891$ \\
\hline The total score of quality of marital life & 27 & 67 & $45.01 \pm 14.750$ \\
\hline
\end{tabular}

AJNPP

Table 3. Mediation test for an indirect relationship using the Bootstrap method

\begin{tabular}{|c|c|c|c|c|c|}
\hline Indirect Pathway & Data & Boot & V & $\begin{array}{l}\text { Lower } \\
\text { Bound }\end{array}$ & $\begin{array}{l}\text { Upper } \\
\text { Bound }\end{array}$ \\
\hline $\begin{array}{c}\text { Illness perception } \rightarrow \text { marital quality } \rightarrow \text { difficulty in emotion } \\
\text { regulation }\end{array}$ & 0.0234 & 0.0231 & 0.0002 & 0.0641 & 0.2021 \\
\hline
\end{tabular}

AJNPP

Table 4. Fitness indices of the research model

\begin{tabular}{cccc}
\hline Indices & Ratio & Limit \\
\hline$\chi^{2}$ & 0.254 & - \\
DF & 1 & - \\
RMSEA & 0.057 & Lower than 0.08 \\
NFI & 0.985 & More than 0.9 \\
NNFI than 0.9 & More than 0.9 \\
CFI & 0.97 & 0.92 & More than 0.9 \\
IFI & 0.90 & More than 0.9 \\
GFI & 0.93 & More than 0.9
\end{tabular}


Table 5. Path coefficients of the direct effect of illness perception on marital life quality

\begin{tabular}{rcccc}
\hline Pathway & Beta & SE & CR & P \\
\hline Illness perception $\rightarrow$ Quality of marital life & -0.361 & 0.015 & -23.421 & 0.005 \\
IIIness perception $\rightarrow$ Difficulty in emotion regulation & 0.257 & 0.035 & 4.400 & 0.003 \\
\hline
\end{tabular}

that patients have about their illness, its complications, and how to cope with it [22]. When a woman develops breast cancer, her perception of this illness plays an important role in her marital functions. The quality of marital life in such patients can be affected by the extent she believes the disease affects her life, how long the illness lasts, how she can control her illness, how effective the treatment is in improving the illness, the extent she has experienced the symptoms of the illness, the extent she is worried about her illness, and the extent it has affected her marital feelings and emotions. Therefore, illness perception can play an important role in the quality of marital life of patients with breast cancer. Negative illness perceptions, such as uncontrollable untreated illness and patient's unawareness about the symptoms have a negative and adverse effect on patient's quality of marital life, leading to a reduction in their marital satisfaction, agreement, and cohesion. Those fail to experience the needed quality of marital life, they face trouble and difficulty in regulating their emotions [23]. Accordingly, the relationship between illness perception and the difficulty in emotion regulation with the mediating role of quality of marital life is significant.

Concerning the relationship between illness perception and quality of marital life, the Leventhal's theory of selfregulation (the common-sense model) expresses two important aspects of the perception of illness: firstly, patient's beliefs about the conditions, in which they are often different from those that the therapist thinks, and secondly, this perception varies from patient to patient. Patients with the same condition may even have very different views regarding their illness. According to the Leventhal's theory, the person's beliefs with a mediating role are moderators when a crisis has arisen. Effective assessment and perception of the illness need understanding the patient's ability to exhibit adaptive behaviors and assessing his success in regulating symptoms of the illness, and subsequently seeking emotional well-being [24]. According to this theory, the beliefs about illness are directly related to the patient's adaptation and behavior. Therefore, the patient's beliefs about her illness can affect his adaptation to breast cancer. Consequently, this adjustment affects the patient's perception of her disability and quality of life. When a patient experiences a higher quality of life, it can also affect her marital life and increase marital agreement, satisfaction, and cohesion. Accordingly, there is a relationship between illness perception and quality of marital life.

Regarding the relationship between illness perception and difficulty in emotion regulation, illness perception is an organized cognitive representation of the patient about her illness. In the Leventhal's theory, diagnosis and the course of the illness are considered. According to this theory, health-related actions are derived from illness perceptions. This model considers healthy behaviors as a result of multifaceted and complex perceptions of the disease. Based on this model, the patient plays a dynamic and active role in illness perception. Patients who believe in the severity of illness consequences (causing problems in his personal life and for others, etc.) and prolonged illness duration are more likely to experience more emotional problems during their illness. Also, those who have made others aware of their illness and are afraid of being ostracized and losing their friends and feel that others avoid facing them are more likely than others to believe in long-term illness. In addition, these people think they have a lower impact on their illness with a lower control over it.

Patients who feel shame and guilt regarding their conditions, and regard themselves as disabled individuals, are more likely to experience emotional states, such as fear of death and aggressiveness concerning their conditions. Also, such patients are not usually aware of their illness and are anxious in dealing with it and believe that their illness will last for a long time. In the Leventhal's self-regulation theory, regulating the illness is based on its type. This theory argues that patients in dealing with illness or a life-threatening factor create a general image and specific belief of the illness and its treatment in their mind that is called illness perception. This perception affects the patient's behavior, adjustment to the illness, management of the illness by the patient, and in general, the outcome of the illness [25].

Women afflicted with breast cancer may experience more emotion dysregulation and treat it inconsistently, which makes it difficult to regulate their emotions, if they do not have an effective and efficient perception of their illness, and believe in their long-term disease and ineffective treatment and follow-up. Accordingly, there is a relation- 
ship between illness perception and difficulty in emotion regulation.

It should be noted that the findings of this study were limited to women with breast cancer in Amol and Babol; thus, caution should be applied in generalizing the results to other women with breast cancer in other cities. Other limitations of this study were data collection using self-report tools; therefore, using other data collection methods, such as using other family members and their physicians will enrich the findings. This study was conducted on a small sample size; however, using a larger sample size can result in different findings from those obtained in the present study. Difficulties in obtaining patients' consent to participate in the research and answer questionnaires were other limitations of the study, as some patients considered doing such research to be unprofitable.

Our statistical population limited to women with breast cancer in Amol and Babol. Therefore, it is recommended to conduct further studies in other cities to approve the relationships obtained and generalize the results further. It is suggested that the researchers test the current research model to predict difficulty in emotion regulation in these patients based on other psychological, emotional, and personality functions. The use of longitudinal studies and other research methods (including qualitative and quantitative combinations) may be more useful to evaluate this study. It is suggested to conduct a qualitative study to investigate the factors affecting the difficulty in emotion regulation of patients with breast cancer. Using simultaneous measurement methods, such as observation and interviews can further highlight the factors affecting the difficulty in regulating the emotion of these patients.

The results and implications of this study can be discussed in both theoretical and practical levels. At the theoretical level, the research findings can help to expand the knowledge, concepts, and models of difficulty in emotion regulation in women with breast cancer by explaining the effect of illness perception with the mediating role of quality of marital life. Also, the results of the present study can be the starting point for future studies to extend the knowledge about the psychological factors causing difficulty in emotion regulation. At the practical level, the findings of this study can be used to design therapeutic and educational programs and interventions in relevant organizations, design interventions by psychologists, counselors, and clinical therapists assisted by health centers, and design couples' training courses to improve emotional problems, such as difficulty emotion regulation in patients suffering from breast cancer.

\section{Conclusion}

The evaluated model had a good fit, which is an important step in understanding the factors affecting the difficulty in emotion regulation in married women with breast cancer. Therefore, it can be useful as a model for designing and developing programs for the prevention of emotional problems in women with breast cancer.

\section{Ethical Considerations}

Compliance with ethical guidelines

All ethical principles were considered in this article. The participants were informed about the purpose of the research and its implementation stages and signed the informed consent. They were also assured of the confidentiality of their information. Moreover, they were allowed to leave the study whenever they wish, and if desired, the results of the research would be available to them.

\section{Funding}

This article was extracted from a PhD. thesis of first author in General Psychology at the Islamic Azad University, Tonekabon Branch and approved by the Ethics Committee of the Islamic Azad University, Tonekabon Branch (Code: IR.IAU.TON.REC.1397.029).

\section{Authors' contributions}

Conceptualization: Raheleh Firouzi; Methodology and supervision: Taher Tizdast; Investigation: Javad Khalatbari; Writing-original draft: Shohreh Ghorban Shiroudi; Writing-review \& editing, funding acquisition, resources: Aall authors.

\section{Conflict of interest}

The authors declared no conflict of interests.

\section{References}

[1] Alacacioglu A, Ulger E, Varol U, Yildiz I, Salman T, Bayoglu V, et al. Depression, anxiety and sexual satisfaction in breast cancer patients and their partners-izmir oncology group study. Asian Pacific Journal of Cancer Prevention. 2014; 15(24):10631-6. [DOI:10.7314/APJCP.2014.15.24.10631] [PMID]

[2] Bai L, Arver B, Johansson H, Sandelin K, Wickman M, Brandberg Y. Body image problems in women with and without breast cancer 6-20 years 
after bilateral risk-reducing surgery-A prospective follow-up study. The Breast. 2019; 44:120-7. [DOI:10.1016/j.breast.2019.01.013] [PMID]

[3] Schlatter MC, Cameron LD. Emotional suppression tendencies as predictors of symptoms, mood, and coping appraisals during AC chemotherapy for breast cancer treatment. Annals of Behavioral Medicine. 2010; 40(1):15-29. [DOI:10.1007/s12160-010-9204-6] [PMID]

[4] Majoor B, Andela C, Quispel C, Rotman M, Dijkstra P, Hamdy N, et al. Illness perceptions are associated with quality of life in patients with fibrous dysplasia. Calcified Tissue International. 2018; 102(1):23-31. [DOI:10.1007/s00223-017-0329-5] [PMID] [PMCID]

[5] Ozdemir D, Tas Arslan F. An investigation of the relationship between social support and coping with stress in women with breast cancer. Psychooncology. 2018; 27(9):2214-9. [DOI:10.1002/pon.4798] [PMID]

[6] Patsou ED, Alexias GT, Anagnostopoulos FG, Karamouzis MV. Physical activity and sociodemographic variables related to global health, quality of life, and psychological factors in breast cancer survivors. Psychology Research and Behavior Management. 2018; 11:371-81. [DOI:10.2147/ PRBM.S170027] [PMID] [PMCID]

[7] Allendorf K, Ghimire DJ. Determinants of marital quality in an arranged marriage society. Social Science Research. 2013; 42(1):59-70. [DOI:10.1016/j.ssresearch.2012.09.002] [PMID] [PMCID]

[8] Umberson D, Williams K, Powers DA, Liu H, Needham B. You make me sick: Marital quality and health over the life course. Journal of Health and Social Behavior. 2006; 47(1):1-16. [DOI:10.1177/0022146511402959] [PMID] [PMCID]

[9] van Leeuwen C, Post MW, van Asbeck FW, van der Woude LH, de Groot $\mathrm{S}$, Lindeman E. Social support and life satisfaction in spinal cord injury during and up to one year after inpatient rehabilitation. Journal of Rehabilitation Medicine. 2010; 42(3):265-71. [DOI:10.2340/16501977-0502] [PMID]

[10] Oh YK, Hwang SY. Impact of uncertainty on the quality of life of young breast cancer patients: Focusing on mediating effect of marital intimacy. Journal of Korean Academy of Nursing. 2018; 48(1):50-8. [DOI:10.4040/ jkan.2018.48.1.50] [PMID]

[11] Taylor EC, O'Neill M, Hughes LD, Moss-Morris R. An illness-specific version of the Revised Illness Perception Questionnaire in patients with atrial fibrillation (AF IPQ-R): Unpacking beliefs about treatment control, personal control and symptom triggers. Psychology \& Health. 2018; 33(4):499-517. [DOI:10.1080/08870446.2017.1373113] [PMID]

[12] Wu X, Lau JT, Mak WW, Gu J, Mo PK, Wang X. How newly diagnosed HIV-positive men who have sex with men look at HIV/AIDS-validation of the Chinese version of the revised illness perception questionnaire. BMC Infectious Diseases. 2018; 18(1):2. [DOI:10.1186/s12879-017-2902-y] [PMID] [PMCID]

[13] Ghio D, Thomson W, Calam R, Ulph F, Baildam EM, Hyrich K, et al. The prioritization of symptom beliefs over illness beliefs: The development and validation of the Pain Perception Questionnaire for Young People. British Journal of Health Psychology. 2018; 23(1):68-87. [DOI:10.1111/ bjhp.12275] [PMID] [PMCID]

[14] Scerri J, Saliba T, Saliba G, Scerri CA, Camilleri L. Illness perceptions, depression and anxiety in informal carers of persons with depression: A cross-sectional survey. Quality of Life Research. 2019; 28(2):451-60. [DOI:10.1007/s11136-018-2009-y] [PMID] [PMCID]

[15] Chou F-Y. Cancer illness perception and self-management of chinese patients. Asia-Pacific Journal of Oncology Nursing. 2019; 6(1):57. [DOI:10.4103/apjon.apjon_56_18] [PMID] [PMCID]
[16] Cunningham ML, Griffiths S, Baillie A, Murray SB. Emotion dysregulation moderates the link between perfectionism and dysmorphic appearance concern. Psychology of Men \& Masculinity. 2018; 19(1):59. [DOI:10.1037/men0000082]

[17] Fillo J, Kamper-DeMarco KE, Brown WC, Stasiewicz PR, Bradizza CM Emotion regulation difficulties and social control correlates of smoking among pregnant women trying to quit. Addictive Behaviors. 2019; 89:104-12. [DOI:10.1016/j.addbeh.2018.09.033] [PMID] [PMCID]

[18] Reffi AN, Pinciotti CM, Darnell BC, Orcutt HK. Trait mindfulness and PTSD symptom clusters: Considering the influence of emotion dysregulation. Personality and Individual Differences. 2019; 137:62-70. [DOI:10.1016/j.paid.2018.08.010]

[19] Busby DM, Christensen C, Crane DR, Larson JH. A revision of the Dyadic Adjustment Scale for use with distressed and nondistressed couples: Construct hierarchy and multidimensional scales. Journal of Marital and Family Therapy. 1995; 21(3):289-308. [DOI:10.1111/j.1752-0606.1995. tb00163.x

[20] Toija AS, Kettunen TH, Leidenius MHK, Vainiola THK, Roine RPA. Effectiveness of peer support on health-related quality of life in recently diag nosed breast cancer patients: A randomized controlled trial. Supportive Care in Cancer. 2019; 27(1):123-30. [DOI:10.1007/s00520-018-4499-0] [PMID] [PMCID]

[21] Bouchard LC, Fisher HM, Carver CS, Kim Y, Antoni MH. Social comparisons predict health-related quality of life and depressive symptoms across the first year of breast cancer treatment. Psychooncology. 2019; 28(2):386-93. [DOI:10.1002/pon.4954] [PMID] [PMCID]

[22] Chang O, Choi E-K, Kim I-R, Nam S-J, Lee JE, Lee SK, et al. Association between socioeconomic status and altered appearance distress, body image, and quality of life among breast cancer patients. Asian $\mathrm{Pa}$ cific Journal Cancer Prevention. 2014; 15(20):8607-12. [DOI:10.7314/ APJCP.2014.15.20.8607] [PMID]

[23] Kugbey N, Meyer-Weitz A, Asante KO. Access to health information health literacy and health-related quality of life among women living with breast cancer: Depression and anxiety as mediators. Patient Education and Counseling. 2019; 102(7):1357-63. [DOI:10.1016/j. pec.2019.02.014] [PMID]

[24] Doege D, Thong MS-Y, Koch-Gallenkamp L, Bertram H, Eberle A, Holleczek $B$, et al. Health-related quality of life in long-term disease-free breast cancer survivors versus female population controls in Germany. Breast Cancer Research and Treatment. 2019; 175(2):499-510. [DOI:10.1007/ s10549-019-05188-x] [PMID]

[25] Kelly DL, Yang GS, Starkweather AR, Siangphoe U, Alexander-Delpech $\mathrm{P}$, Lyon DE. Relationships among fatigue, anxiety, depression, and pain and health-promoting lifestyle behaviors in women with early-stage breast cancer. Cancer Nursing. 2019; 11(3):45-59. 
This Page Intentionally Left Blank 